\title{
EFECTO DE UN PROGRAMA DE ACTIVIDAD FÍSICA DE MODERADA A VIGOROSA DE DIEZ MESES SOBRE EL VO,MÁX Y EL PORCENTAJE DE GRASA CORPORAL EN NIÑOS CON SOBREPESO Y OBESIDAD
}

\section{EFFECT OF A TEN-MONTH MODERATE TO VIGOROUS PHYSICAL ACTIVITY PROGRAM ON $V_{2}$ MAX AND THE PERCENTAGE OF BODY FAT IN OVERWEIGHT AND OBESE CHILDREN}

\author{
Javier Arturo Hall-López ', (0000-0002-7449-2912) \\ Paulina Yesica Ochoa-Martínez ${ }^{l}$, \\ Adrián Zamudio Bernal ${ }^{1}$, \\ Ricardo Sánchez León ', \\ Luis Gerardo Uriarte Garza ", \\ Bartolomé Jesús Almagro ${ }^{2}$, \\ José Moncada-Jiménez ${ }^{3,4}$ (0000-0001-9807-5163) \\ Pedro Sáenz-López Buñuel ${ }^{2}$
}

${ }^{1}$ Facultad de Deportes, Universidad Autónoma de Baja California, México. ${ }^{2}$ Departamento de Didácticas Integradas, Universidad de Huelva, España. ${ }^{3}$ Escuela de Educación Física y Deportes, Universidad de Costa Rica, Costa Rica. ${ }^{4}$ Centro de Investigación en Ciencias del Movimiento Humano, Universidad de Costa Rica, Costa Rica. pochoa@uabc.edu.mx

\section{Resumen}

Objetivo: Evaluar el efecto de un programa de actividad física con intensidad de moderada a vigorosa bajo el modelo CATCH sobre el porcentaje de grasa corporal y capacidad aeróbica en niños con sobrepeso u obesidad. Método: Veintiséis niños (15 hombres y 11 mujeres) (edad $=9.4 \pm 0.3$ años), diagnosticados con sobrepeso u obesidad por su índice de masa corporal, participaron en un programa de actividad física con intensidad moderada a vigorosa bajo el modelo de $\mathrm{CATCH}, 2$ veces por semana por un periodo de 10 meses. El programa estuvo compuesto por ejercicios de intensidad moderada a vigorosa $(56 \%)$ y fue evaluado por el sistema para observar el tiempo de instrucción de actividad física (SOFIT). Antes y después del programa se evaluó el porcentaje de grasa corporal con la ecuación de Slaughter et al. (1988), valorando los pliegues cutáneos de tríceps y pantorrilla. La capacidad aeróbica se evaluó con el test Course Navette de 20 metros calculando el consumo máximo de oxígeno $\left(\mathrm{VO}_{2}\right.$ máx) por la ecuación de Léguer et al. (1984). Resultados: Se encontró una reducción significativa en el porcentaje de grasa corporal $(\Delta \%=-2.4, p=0.022)$ y un aumento significativo en el $\mathrm{VO}_{2}$ máx $(\Delta \%=11.3, \mathrm{p} \leq 0.001)$. Conclusión: La actividad física con intensidad de moderada a vigorosa bajo el modelo de CATCH fue capaz de mejorar la capacidad aeróbica y reducir el porcentaje de grasa corporal en niños con sobrepeso u obesidad.

Palabras clave: Obesidad infantil, tejido graso, ejercicio aeróbico. 
URL: http://www.revistas.una.ac.cr/mhsalud
Javier Arturo Hall-López, Paulina Yesica Ochoa-Martínez, Adrián Zamudio Bernal, Ricardo Sánchez León, Luis Gerardo Uriarte Garza, Bartolomé Jesús Almagro, José Moncada-Jiménez, Pedro Sáenz-López Buñuel

\begin{abstract}
Objective: Evaluate the effect of a moderate to vigorous physical activity program under the CATCH model on the body fat percentage and aerobic capacity in overweight or obese children. Method: Twenty-six children (15 males and 11 females) (age $9.4 \pm 0.3$ years) diagnosed as overweight or obese from their body mass index (BMI) participated in a moderate to vigorous physical activity program under the CATCH model, 2 times a week for a 10-month period, comprised of moderate to vigorous exercises (56\%) and evaluated using the System for Observing Fitness Instruction Time (SOFIT). The body fat percentage was evaluated before and after the program using the equation by Slaughter et al., 1988 taking the anthropometrics measurements of triceps and media calf skin folds. The aerobic capacity (maximum oxygen uptake- $\mathrm{VO}_{2 \max }$ ) was determined through the 20-meter Multi-Stage Fitness Test using the equation proposed by Léguer et al., 1984. Results: The results showed a significant decrease in the body fat percentage $(\Delta \%=-2.4, \mathrm{p}=0.022)$ and a significant increase in $\mathrm{VO}_{2} \max (\Delta \%=11.3, \mathrm{p} \leq 0.001)$. Conclusion: Having moderate to vigorous physical activity under the CATCH model improved aerobic capacity and reduced the body fat percentage in overweight or obese children.
\end{abstract}

Keywords: Pediatric Obesity, Adipose Tissue, Aerobic Exercise.

\title{
Introducción
}

La obesidad es un problema de salud creciente que afecta cada vez a más niños en el mundo, sin importar género, grupo étnico o nivel socioeconómico. De acuerdo con datos del International Obesity Task Force (IOTF), a nivel mundial el $20 \%$ de los niños y adolescentes presentan estas patologías (Kelly et al., 2013). En México, la Encuesta Nacional de Salud y Nutrición, ENSANUT, (INSP, 2012) reportó en un $34.4 \%$ la prevalencia combinada de sobrepeso y obesidad en niños de edad escolar entre 5 y 11 años (Janssen et al., 2013). El problema de la obesidad infantil radica en su alta prevalencia y su relación con el riesgo cardiovascular que repercute en la mortalidad y la calidad de vida de la población (Ebbeling y Ludwig, 2010).

Aunque la obesidad tiene un origen multifactorial, los cambios en el estilo de vida actual han incluido la adopción de alimentos altos en energía, densos en los contenidos de grasas saturadas y carbohidratos refinados, así como una disminución de la actividad física. Estos factores provocan un desequilibrio en el balance energético del organismo, el cual almacena grasa excesivamente como potencial fuente de energía y como consecuencia desarrolla la obesidad (Broyles et al., 2010). Se conoce que la masa grasa visceral es un factor determinante de la resistencia a la insulina, lo cual a su vez está asociado al deterioro progresivo que conduce a la diabetes y enfermedades cardiovasculares (Rank et al., 2013). Asimismo, existe evidencia que indica que la falta de actividad física es un factor de riesgo para la aparición del síndrome metabólico (Blanchard et al., 2013). Los niños con sobrepeso y obesidad son un sector de la población al que se le debe poner especial atención, ya que cada día hay más niños obesos con mayores facilidades y posibilidades consumo de alimentos hipercalóricos (Kelly et al., 2013).

Broyles et al. (2010) han demostrado una alta correlación entre la obesidad infantil, los padres obesos y los hábitos de vida; es decir, un niño tendrá mayor posibilidad de ser obeso 
URL: http://www.revistas.una.ac.cr/mhsalud
Javier Arturo Hall-López, Paulina Yesica Ochoa-Martínez, Adrián Zamudio Bernal, Ricardo Sánchez León, Luis Gerardo Uriarte Garza, Bartolomé Jesús Almagro, José Moncada-Jiménez, Pedro Sáenz-López Buñuel

si sus padres son obesos. Por este motivo, se deben buscar formas de inducir hábitos de vida saludables que incluyan alimentación balanceada y actividad física en las familias de los niños con riesgo de desarrollar sobrepeso y obesidad, ya que esto representa un aporte significativo en la prevención de padecimientos asociados con este problema de salud (Ebbeling y Ludwig, 2010). Por otro lado, el solo hecho de cambiar los hábitos de actividad física infantil provoca cambios importantes y quizá de mayor impacto en la salud del individuo en el largo plazo (Blanchard et al., 2013). Los niños son más sensibles a los intentos de modificar su peso corporal que los adultos, en especial en cuanto a fenómenos de distribución y porcentaje de grasa corporal (Tailor et al., 2007; Lizana et al., 2011; Hall et al., 2013).

La prueba de campo denominada test Course Navette de 20 metros, que ha sido evaluada en 1142026 niños y adolescentes de más de 50 países, ha sido comúnmente utilizada en el ámbito de escolar para determinar el parámetro fisiológico denominado consumo máximo de oxígeno ( $\mathrm{VO}_{2}$ máx). Este parámetro se define numéricamente como la velocidad y la capacidad en la que una persona respira aire del medio ambiente, lo transporta por el sistema respiratorio y cardiovascular, y metaboliza el oxígeno $\left(\mathrm{O}_{2}\right)$ como fuente de energía en las células musculares al realizar actividad física (Welk et al., 2011). En el ámbito de la salud, resultados de estudios longitudinales muestran claramente que niños con bajos valores de $\mathrm{VO}_{2}$ máx evaluado mediante el test Course Navette de 20 metros presentan obesidad, enfermedades metabólicas y cardiovasculares (Ruiz et al., 2011). Para solucionar esta problemática, se han realizado múltiples programas de intervención exitosos para prevenir el sobrepeso y la obesidad infantil, tal es el caso del programa Coordinated Approach to Child Health (CATCH), llevado acabo por un equipo de investigación de cuatro universidades: University of California at San Diego, University of Minnesota, Tulane University y University of Texas Health Science Center at Houston.

El programa CATCH ha sido extensamente evaluado en más de 80 publicaciones científicas. Una investigación longitudinal abarcó 96 escuelas (56 de intervención y 40 control), en cuatro estados de Estados Unidos (California, Luisiana, Minnesota y Texas), y se incluyeron alrededor de 5100 estudiantes de 3ro a 5to grado de diversos grupos étnicos y culturales. Para ejecutar el programa CATCH en las escuelas, se consideraron muchos componentes para la promoción de la salud, incluyendo un programa para la disminución del consumo de grasa, grasa saturada y sodio en la dieta de los niños, aumentar la cantidad de actividad física y prevenir el fumado (Perry et al., 1990). El programa también incluyó modificaciones en el ambiente, en donde el componente de actividad física se enfocó en que la educación física se proporcionara cinco veces por semana y los maestros de educación física fueran instruidos para involucrar a los niños en actividad física e incrementar la actividad física de moderada a vigorosa intensidad al menos el 50 \% del tiempo de la clase (Kelder et al., 2003). El programa CATCH tuvo resultados positivos en producir al menos cambios en la dieta y conductas relacionadas a la actividad física, en donde los estudiantes que participaron en el programa CATCH consumieron menos grasa y participaron en más actividades físicas fuera de la escuela. Tres años después de la intervención, se mostró una sostenibilidad al observar que el bajo consumo de grasa y los altos niveles de 

Adrián Zamudio Bernal, Ricardo Sánchez León, Luis Gerardo Uriarte Garza, Bartolomé Jesús Almagro, José Moncada-Jiménez, Pedro Sáenz-López Buñuel

actividad física fueron mayores de los estudiantes participantes en comparación con los niños del grupo control (Osganian et al., 2003). Estos resultados posibilitaron su implementación como política pública de salud en el medio escolar.

El sobrepeso y la obesidad infantil requerirán que los sistemas de atención de salud pública y educativa se adapten de manera específica y eficiente para tratar estas enfermedades dentro de este grupo de edad (Janssen et al., 2012). Con base en esta fundamentación teórica, es importante fomentar actividad física adecuada para las necesidades del niño con esta patología. Por lo anterior, el objetivo de la presente investigación fue evaluar el efecto de un programa de actividad física con intensidad de moderada a vigorosa bajo el modelo CATCH sobre el porcentaje de grasa corporal y la capacidad aeróbica en niños con sobrepeso y obesidad.

\section{Metodología}

\section{Participantes}

Este estudio se llevó a cabo bajo un diseño metodológico cuasi-experimental con muestreo no probabilístico por conveniencia, donde la variable independiente (programa de actividad física con intensidad de moderada a vigorosa bajo el modelo de CATCH en niños con sobrepeso u obesidad) fue manipulada para medir su efecto sobre las variables dependientes (porcentaje de grasa corporal y capacidad aeróbica), con el propósito de determinar el grado de cambio producido por el tratamiento estableciendo una relación causa-efecto. Se atendieron los principios éticos de investigación en seres humanos de la Declaración de Helsinki. El proyecto de investigación fue sometido a evaluación ante el comité del programa de la 18va convocatoria interna y aprobado por la Coordinación de Posgrado en Investigación de la Universidad Autónoma de Baja California (Protocolo con clave 149/2/C/9/18). La institución en la cual se llevó a cabo esta investigación fue la Escuela Primaria Teniente Andrés Arreola, de la Ciudad de Mexicali, Baja California, México, con niños de cuarto grado de educación primaria.

Participaron 26 niños con sobrepeso u obesidad, 15 niños y 11 niñas, con una edad promedio de $9.4 \pm 0.3$ años. Para la selección de los participantes, se utilizaron los siguientes criterios de inclusión: a) participar de manera voluntaria, b) haber nacido en el año 2006, c) estar clasificados con un estado nutricional de sobrepeso u obesidad, d) no haber participado por lo menos tres meses antes en un programa de ejercicio físico sistemático. Los únicos criterios de exclusión para participar en el estudio fueron los siguientes: que los niños no quisieran participar o que estuvieran diagnosticados con cualquier patología de condición aguda o crónica que pudiera impedir realizar ejercicio físico.

\section{Instrumentos}

Para determinar el peso y la estatura corporal se utilizó una báscula con estadiómetro (Seca, modelo 220, Hamburgo, Alemania) con precisión de $100 \mathrm{~g}$ y $0.1 \mathrm{~cm}$, respectivamente. Las mediciones 
URL: http://www.revistas.una.ac.cr/mhsalud
Javier Arturo Hall-López, Paulina Yesica Ochoa-Martínez, Adrián Zamudio Bernal, Ricardo Sánchez León, Luis Gerardo Uriarte Garza, Bartolomé Jesús Almagro, José Moncada-Jiménez, Pedro Sáenz-López Buñuel

se realizaron siguiendo los estándares de la Sociedad para el Avance de la Cineantropometría, ISAK, (Marfell-Jones, Olds, Stewart y Carter, 2006). Con los valores de peso y estatura, se calculó el índice de masa corporal $(\mathrm{IMC})$ de la siguiente manera: $\mathrm{IMC}=$ peso $(\mathrm{kg}) /$ talla $\left(\mathrm{m}^{2}\right)$.

El porcentaje de grasa corporal se determinó mediante las mediciones antropométricas de pliegue de tríceps $(\mathrm{mm})$ y pantorrilla $(\mathrm{mm})$ del lado derecho del cuerpo utilizando un Skinfold Caliper Slim Guide y siguiendo lineamientos establecidos en ISAK (Marfell-Jones et al., 2006). Para el cálculo de la variable se utilizó la fórmula validada por Slaughter et al. (1988) para hombres $=0.735$ (pliegue de tríceps $[\mathrm{mm}]+$ pliegue de pantorrilla $[\mathrm{mm}])+1.0$ y para mujeres $=$ 0.610 (pliegue de tríceps $[\mathrm{mm}]+$ pliegue de pantorrilla $[\mathrm{mm}]$ ) +5.1 .

La capacidad aeróbica se determinó con la prueba de campo Course Navette de 20 metros, la cual ha sido validada en niños y adolescentes de 6 a 17 años de edad $(r=0.70)$ (Léger et al., 1984). La prueba establece la capacidad aeróbica midiendo de manera indirecta por medio del $\mathrm{VO}_{2}$ máx.

Para conocer la intensidad de la actividad física durante programa bajo el modelo CATCH se utilizó el Sistema para Observar el Tiempo de Instrucción de Actividad Física (SOFIT, por sus siglas en inglés de System for Observing the Fitness and Instruction Time) (McKenzie et al., 1992). Con este método, mediante observación directa, se cuantificó la actividad física de moderada a vigorosa. Se eligieron al azar cuatro participantes en cada sesión, en una secuencia rotatoria de 12 intervalos durante 20s cada uno, a la velocidad de un audio del instrumento SOFIT que el evaluador reprodujo en un aparato MP3 Samsung YP-U6AB. Las observaciones se repitieron durante toda la sesión. SOFIT fue validado por medio de monitoreo los latidos del corazón y a través del uso de acelerómetros.

\section{Procedimientos}

Para conocer el estado nutricional de cada participante se utilizó el IMC y el valor obtenido se clasificó de acuerdo con su percentil (P) tomando en consideración la edad y el género del participante. Se definió como sobrepeso un valor entre el $\mathrm{P}_{85 \%} \mathrm{y}<\mathrm{P}_{95 \%}$ y como obesidad, un valor $>\mathrm{P}_{95 \%}$ a partir de las referencias del Center for Disease Control and Prevention (CDC), por medio del software Epi Info, versión 3.5.1. Posteriormente, a cada participante se le midió el porcentaje de grasa y realizó la prueba de Course Navette. Se estableció una marca de inicio y fin de una distancia de 20 metros en el suelo de forma transversal, para que los niños se desplazaran corriendo en esa distancia bajo la instrucción de un ritmo dado por un sonido emitido de una grabación, con una velocidad de desplazamiento inicial $8.5 \mathrm{~km} / \mathrm{h}$ que se incrementó de manera progresiva cada minuto de acuerdo al sonido. La prueba finalizó cuando los participantes no podían terminar los desplazamientos de carrera con el ritmo y no alcanzaban a llegar a la línea o se retiraban voluntariamente de la prueba debido a la fatiga. Una vez terminada la prueba el evaluador anotó los niveles y vueltas realizadas por los participantes y de acuerdo a estos valores se estimó el VO máx con la siguiente fórmula: $\mathrm{VO}_{2}$ máx $\left(\mathrm{ml} \cdot \mathrm{kg}^{-1} \cdot \mathrm{min}^{-1}\right)=31.025+3.238(\mathbf{V})-3.248(\mathbf{E})+0.1536(\mathbf{V E})$; en donde $\mathbf{V}$ es la velocidad del último nivel alcanzado durante el test en $\mathrm{km} / \mathrm{h}(\mathrm{V}=8+0.5 \mathrm{x}$ último nivel alcanzado) y $\mathbf{E}$ representa la edad en años. La evaluación inicial y final de las variables se realizaron tres días previos y tres días posteriores al inicio y final del programa CATCH. 


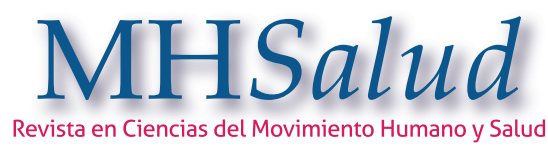

URL: http://www.revistas.una.ac.cr/mhsalud
Javier Arturo Hall-López, Paulina Yesica Ochoa-Martínez, Adrián Zamudio Bernal, Ricardo Sánchez León, Luis Gerardo Uriarte Garza, Bartolomé Jesús Almagro, José Moncada-Jiménez, Pedro Sáenz-López Buñuel

El programa de actividad física estuvo compuesto por dos sesiones a la semana de $50 \mathrm{~min}$, que se dividieron en 5 min de calentamiento, 40 min de componente aeróbico y 5 min de vuelta a la calma (cool-down) con estiramientos y movimientos que redujeran de manera progresiva la frecuencia cardiaca. Los participantes completaron 10 meses del programa de actividad física con intensidad de moderada a vigorosa bajo el modelo CATCH. Este modelo se basó en la planificación efectiva al administrar las sesiones de actividad física: se dieron instrucciones claras y sencillas con una supervisión activa, cada participante contó con su material, se evitaron las largas filas, se utilizaron juegos de corto tiempo, se trató de evitar enfocarse en los estudiantes más destacados, se dio a los estudiantes muchas oportunidades de participación y práctica, se evitaron acciones de eliminación y se limitó la competencia.

Para desarrollar las actividades, se tuvo como apoyo didáctico para el profesor un fichero de actividades que contenía información concisa e instrucciones para que se implementaran en la clase. Las fichas fueron organizadas, escritas y presentadas en un formato simple y claro para facilitar la planificación. Con base en lo anterior, las estrategias didácticas proporcionaban elementos pedagógicos de apoyo docente para que el profesor impartiera la actividad física con intensidad de moderada a vigorosa por lo menos el $50 \%$ del tiempo de la clase. Para determinar la intensidad de la actividad física se usaron códigos para clasificar los niveles de actividad, los cuales permitieron estimar la energía utilizada asociada con la actividad física en cinco categorías: 1) acostado, 2) sentado, 3) de pie, 4) caminando, y 5) muy activo, que corresponde a correr o a cuando el sujeto realiza una actividad física similar o superior a correr. A partir de estas categorías se determinó la actividad física de moderada a vigorosa sumando porcentualmente los códigos 4) caminando y 5) muy activo del total del tiempo de las sesiones. Dos evaluadores fueron capacitados siguiendo el estándar de protocolo de SOFIT y se evaluó el $100 \%$ de las observaciones con una alta confiabilidad (índice Kappa $=0.083)($ Szklo, 2007).

\section{Análisis estadístico}

Solo se tomaron en cuenta para el análisis estadístico a los sujetos que asistieron al $95 \%$ de las sesiones del programa. El análisis estadístico se realizó mediante el Paquete Estadístico para las Ciencias Sociales (SPSS), versión 21.0 para Windows (IBM Corporation, New York, USA). Se obtuvieron estadísticas descriptivas de las variables y los porcentajes de cambio $(\Delta \%)$ ( $^{\text {Promedio }}{ }_{\text {post-test }}-$ Promedio $\left._{\text {pre-test }}\right] /$ Promedio $\left._{\text {pre-test }}\right) \times 100$. Se utilizó la prueba $t$-Student pareada, de medidas repetidas o de grupos relacionados para comparar las diferencias en los promedios de las variables dependientes. Previamente se realizó una evaluación de normalidad con el test Shapiro-Wilk. El valor de significancia se estableció a priori en $\mathrm{p} \leq 0.05$.

\section{Resultados}

La estadística descriptiva de los sujetos evaluados se muestra en la tabla 1. Se encontró que la actividad física moderada a vigorosa fue en promedio de $56 \%$ durante todas las sesiones. En la figura 1 se muestra el porcentaje de grasa inicial de los participantes fue de $28.7 \pm 5.3 \%$ y el final fue de $28.0 \pm 5.1 \%$. El $\mathrm{VO}_{2}$ máx inicial fue de $32.6 \pm 2.1 \mathrm{ml} \cdot \mathrm{kg}^{-1} \cdot \mathrm{min}^{-1} \mathrm{y}$ el final de $36.3 \pm$ 
$2.8 \mathrm{ml} \cdot \mathrm{kg}^{-1} \cdot \mathrm{min}^{-1}$. Se encontró una reducción significativa en el porcentaje de grasa corporal $(\Delta \%$ $=-2.4, \mathrm{p}=0.022)$ y un aumento significativo en el $\mathrm{VO}_{2}$ máx $(\Delta \%=11.3, \mathrm{p} \leq 0.001)$.

Los cambios en la capacidad aeróbica al participar en el programa actividad física con intensidad de moderada a vigorosa bajo el modelo de CATCH en niños con sobrepeso u obesidad se pueden observar en la figura $2(\mathrm{p}=0.000)$.

Tabla 1. Media y desviación estándar $(\mathrm{M} \pm \mathrm{DE})$ y valores de normalidad de las variables de porcentaje de grasa corporal y capacidad aeróbica.

\begin{tabular}{lcccc}
\hline \multirow{2}{*}{ Variables } & \multicolumn{2}{c}{ Evaluación inicial $(\mathbf{n}=\mathbf{2 6})$} & \multicolumn{2}{c}{ Evaluación Final $(\mathbf{n}=\mathbf{2 6})$} \\
\cline { 2 - 5 } & $\mathbf{M} \pm \mathbf{D E}$ & Test Shapiro-Wilk & $\mathbf{M} \pm \mathbf{D E}$ & Test Shapiro-Wilk \\
\hline Grasa corporal $(\%)$ & $28.7 \pm 5.3$ & .046 & $28.0 \pm 5.1$ & .117 \\
$\mathrm{VO}_{\text {2máx }}\left(\mathrm{ml} \cdot \mathrm{kg}^{-} \cdot \mathrm{min}^{-1}\right)$ & $32.6 \pm 2.1$ & .691 & $36.3 \pm 2.8$ & .836 \\
\hline
\end{tabular}

Nota: Los valores presentados de los sujetos evaluados son media y desviación estándar $( \pm)$ del porcentaje de grasa corporal, determinadas por la ecuación de Slaughter et al. (1988), y capacidad aeróbica, determinada por la ecuación de Leguer et al. (1984).

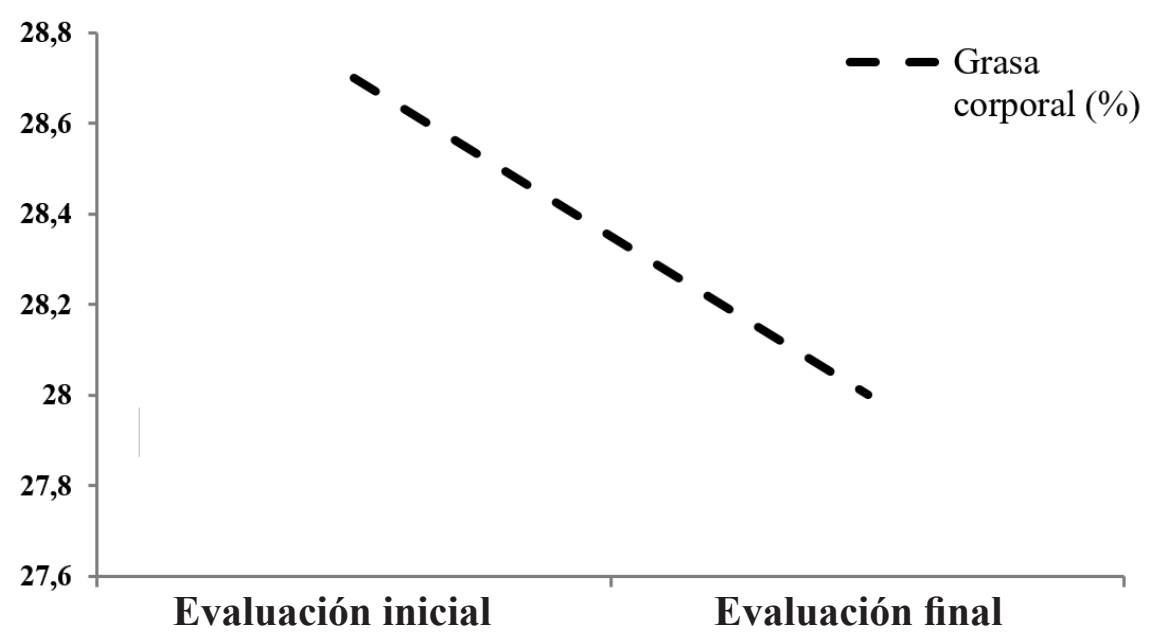

Figura 1. Cambios en el porcentaje de grasa corporal en 10 meses, al participar en el programa de actividad física con intensidad de moderada a vigorosa bajo el modelo de CATCH en niños con sobrepeso u obesidad ( $\mathrm{n}=26)$.

Nota: El cálculo de la igualdad de la varianza fue realizado mediante el test $t$ Student para muestras relacionadas y el del porcentaje de grasa corporal fue determinado por la ecuación de Slaughter et al. (1988): \%=0.735(pliegue de tríceps $[\mathrm{mm}]+$ pliegue de pantorrilla $[\mathrm{mm}])+1.0 \mathrm{y}$, en mujeres, $\%=0.610$ (pliegue de tríceps $[\mathrm{mm}]+$ pliegue de pantorrilla $[\mathrm{mm}])+5.1$. Este cálculo fue realizado antes y después de 10 meses de la participación de niños con sobrepeso u obesidad ( $\mathrm{n}=26)$ en el programa de actividad física con intensidad de moderada a vigorosa bajo el modelo de $\mathrm{CATCH}(\mathrm{p}=0.022)$. 


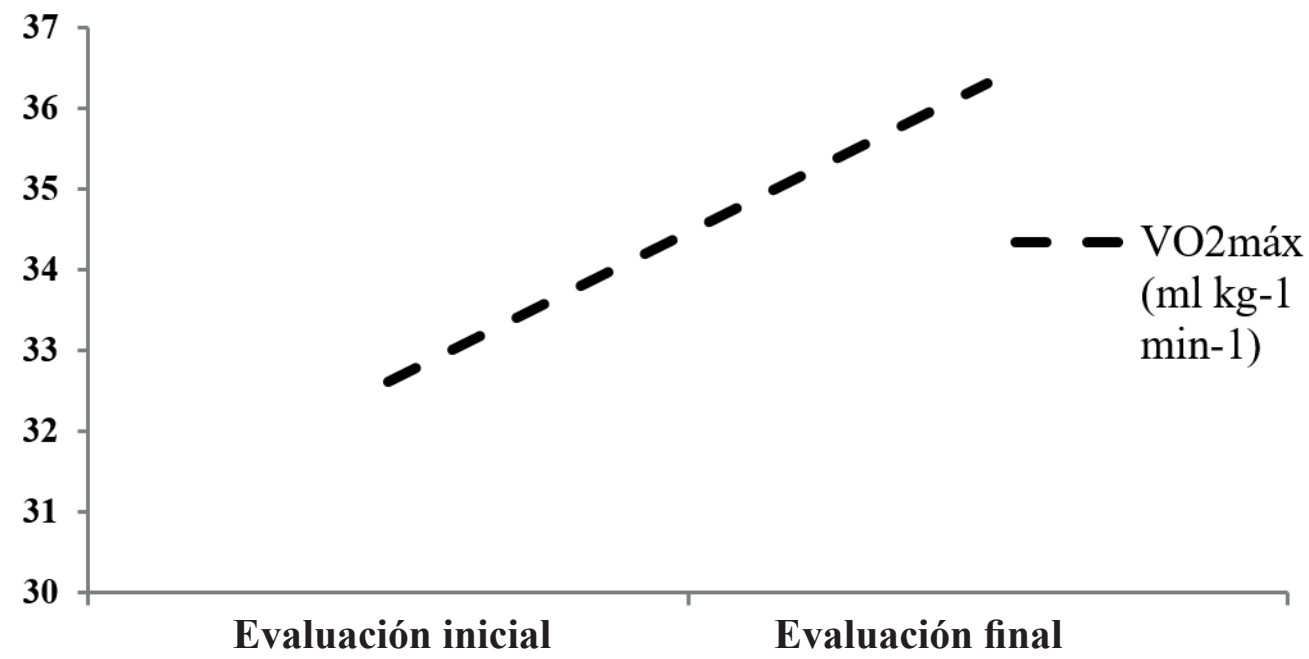

Figura 2. Cambios en la capacidad aeróbica en 10 meses, al participar en el programa de actividad física con intensidad de moderada a vigorosa bajo el modelo de CATCH en niños con sobrepeso u obesidad ( $n=26)$.

Nota: El cálculo de la igualdad de la varianza fue realizado mediante el test $t$ Student para muestras relacionadas. La capacidad aeróbica fue determinada mediante el test Course Navette de 20 metros (Léger, 1984) y estimada con la fórmula VO2máx $=31.025+3.238 \mathrm{~V}-3.248 \mathrm{E}+0.1536 \mathrm{VE}$ ( $\mathrm{V}$ es la velocidad del último nivel alcanzado durante el test en $\mathrm{Km} / \mathrm{h}(\mathrm{V}=8+0.5 \mathrm{x}$ último nivel alcanzado) y E representa la edad en años. Este cálculo fue realizado antes y después de 10 meses de la participación de niños con sobrepeso u obesidad $(n=26)$ en el programa de actividad física con intensidad moderada a vigorosa bajo el modelo de $\mathrm{CATCH}(\mathrm{p}=0.000)$.

\section{Discusión}

El objetivo del estudio fue evaluar el efecto de un programa de actividad física con intensidad de moderada a vigorosa bajo el modelo de CATCH sobre el porcentaje de grasa corporal y la capacidad aeróbica en niños con sobrepeso y obesidad. El principal resultado de la investigación fue que 10 meses de participación de los niños diagnosticados con obesidad - de acuerdo con la edad y género por su índice de masa corporal (IMC) - mejoran de manera significativa el $\mathrm{VO}_{2}$ máx y reducen modestamente el porcentaje de grasa corporal. Estos hallazgos son positivos, pues en investigaciones previas (Ruiz et al., 2011) se ha observado de manera proporcional valores altos de $\mathrm{VO}_{2}$ máx en niños con la presencia en menor medida de obesidad, enfermedades metabólicas y cardiovasculares. En otra investigación llevada a cabo por Welk et al. (2011), se demostraron mejoras significativas en la capacidad aeróbica. En ese estudio se evaluó la capacidad aeróbica en niños con sobrepeso y obesidad, a quienes se les sometió a un programa de ejercicio físico, educación física, actividad física o iniciación deportiva durante seis meses. Se encontró en promedio un aumento de $4.5 \mathrm{ml} \cdot \mathrm{kg}^{-1} \cdot \mathrm{min}^{-1}$ en el $\mathrm{VO}_{2}$ máx. 
URL: http://www.revistas.una.ac.cr/mhsalud
Javier Arturo Hall-López, Paulina Yesica Ochoa-Martínez, Adrián Zamudio Bernal, Ricardo Sánchez León, Luis Gerardo Uriarte Garza, Bartolomé Jesús Almagro, José Moncada-Jiménez, Pedro Sáenz-López Buñuel

En el presente estudio, la prueba de campo denominada test Course Navette de 20 metros para la evaluación de la capacidad aeróbica fue propuesta por ser un instrumento válido en niños y adolescentes de 6 a 17 años de edad, además de ser de fácil aplicación, económica, confiable y que proporciona información relacionada con la salud de los niños (Léger et al., 1984; Welk et al., 2011). Los valores encontrados en relación con la capacidad aeróbica fueron consistentes con otros estudios longitudinales realizados en niños obesos que utilizaron actividad física de intensidad moderada a vigorosa (Huang et al., 2012). Por otra parte, al atender el diseño de sesiones CATCH se enfatizó el trabajo aeróbico al realizarse con frecuencia de dos sesiones por semana. Stanley et al. (2013) mencionan que para obtener mejores adaptaciones biológicas en la capacidad aeróbica se requieren al menos tres sesiones por semana y por lo menos seis meses de práctica de ejercicio físico; sin embargo, esta discrepancia al encontrar diferencias significativas en nuestro estudio la asumimos en cuanto al tiempo de intervención total.

En una investigación realizada en el mismo contexto mexicano, en dos escuelas públicas donde los estudiantes realizaron una rutina de actividad física durante 16 semanas, se encontraron disminuciones en la presión sistólica, la concentración de triglicéridos y colesterol total, pero no hubo cambios en el IMC, circunferencia de cintura e insulina sérica (Perichart-Perera et al., 2008). De igual manera, en otro estudio se evaluó el impacto de una intervención de 18 meses para la prevención de obesidad en escolares de $4^{\circ}$ y $5^{\circ}$ grado basada en el modelo ecológico en conductas saludables; sin embargo, a pesar del tiempo de intervención, la prevalencia de obesidad no se modificó (Safdie et al., 2013). Estos resultados parcialmente contradicen a las investigaciones donde se encontró disminución significativa en el IMC al intervenir niños mexicanos de 6 a 10 meses (Bacardi-Gascon et al., 2012; Elizondo-Montemayor et al., 2013). Cabe destacar que los estudios anteriores enfatizaron su intervención en la alimentación, la cual proporciona la mayor influencia en los cambios de porcentaje de grasa corporal (Gonzalez et al., 2012).

En el ámbito de la cultura física ha sido ampliamente recomendado que los profesionales de la actividad física y la salud tengan competencias en la evaluación del porcentaje de grasa corporal (Lizana et al., 2011; Hall et al., 2013), dado que se requiere interpretar los resultados en niños y padres y pensar cómo lograr mantener la salud a través de la modificación del estilo de vida (actividad física y alimentación). De igual manera, deben saber interpretar la información que se obtiene sobre los cambios en la composición corporal (Slaughter et al., 1998; Tailor et al., 2010; Welk et al., 2011).

El análisis sobre la intensidad de la actividad física en la presente investigación propició estrategias didácticas y elementos pedagógicos en los que el profesor impartió la actividad física con intensidad de moderada a vigorosa por lo menos el $50 \%$ del tiempo de la clase. Otras investigaciones realizadas en el contexto de la educación física en México han evaluado la intensidad de la actividad física de moderada a vigorosa y refirieron una variabilidad en el índice de actividad física moderada y vigorosa de 38.2 \% (Pérez Bonilla, 2009), 29.2 \% (JenningsAburto et al., 2009), 52 \% (Gharib et al., 2015) y 41 \% (Hall-López, Ochoa, González, et al., 2017), 37.9 (Hall-López, Ochoa, Zúñiga, et al., 2017). En el contexto de estas investigaciones, en 

Adrián Zamudio Bernal, Ricardo Sánchez León, Luis Gerardo Uriarte Garza, Bartolomé Jesús Almagro, José Moncada-Jiménez, Pedro Sáenz-López Buñuel

la clase de educación física se percibió la falta de material didáctico para tener más oportunidad de participar, una gran cantidad de tiempo en que los alumnos permanecieron parados mientras el profesor organiza al grupo para participar, largas filas para tener la oportunidad de participar y los tiempos de transición entre las actividades fueron muy largos. Por otra parte, otra investigación donde se midió el tiempo efectivo de la clase de educación física mediante sensores de movimiento resultó en 46.1 \% de actividad física de moderada a vigorosa (Flores et al., 2017).

Por lo anterior, sería propicio combinar los objetivos y metas del programa de educación física mexicano en educación básica y las recomendaciones establecidas por el National Association for Sport and Physical Education (NASPE). Esta institución menciona que las clases de educación física, al ser impartidas por los profesores, deben ser diseñadas para que el estudiante participe en actividades que por lo menos tengan una intensidad de moderada a vigorosa por arriba del $50 \%$ del tiempo de la clase; es decir, que estén activos con un gasto energético similar a caminar o correr (NASPE, 2009; Banville, 2006) y de esa manera coadyuvar para el beneficio de la salud de los niños y jóvenes. La Organización Mundial de la Salud (OMS), por su parte, recomienda que los niños y jóvenes de 5 a 17 años deben invertir como mínimo 60 minutos diarios en actividades físicas de intensidad moderada a vigorosa y que la actividad física diaria debería ser, en su mayor parte, aeróbica, incorporando como mínimo tres veces por semana actividades vigorosas que refuercen en particular los músculos y huesos (OMS, s.f.), dando mayor énfasis a la recomendación de actividad física en niños con sobrepeso y obesidad donde existe mayor incidencia de enfermedades crónicas no trasmisibles (Broyles et al., 2010).

La presente investigación presenta limitaciones al no evaluar un grupo control dentro del diseño metodológico. Además, no se estratificaron los resultados por género debido a la poca cantidad de sujetos de estudio. Esto habría posibilitado un aumento y un mayor poder al análisis de la estadística inferencial al mostrar los resultados y, consecuentemente, habría permitido extrapolar los resultados obtenidos a sujetos con características similares. Por lo anterior, en el futuro sería importante realizar más investigaciones que clarifiquen las posibilidades de efecto sobre las variables estudiadas con el propósito de tener resultados que sirvan de referencia para la planeación, ejecución y evaluación de intervenciones orientadas a prevenir y minimizar el problema de obesidad infantil y que, en consecuencia, sirvan a los investigadores y profesionales de la actividad física y salud que trabajan en torno a esta población para comprender dicha problemática de una manera más apropiada y tener mayores elementos para una mejor atención.

\section{Conclusiones}

En conclusión, un programa de 10 meses de actividad física con intensidad de moderada a vigorosa bajo el modelo CATCH mejora significativamente la capacidad aeróbica y reduce modestamente la adiposidad en niños con sobrepeso y obesidad. Sin embargo, en el futuro es importante realizar más investigaciones que clarifiquen las posibilidades de efecto del ejercicio físico en la grasa corporal en niños con las patologías de sobrepeso y obesidad. 
URL: http://www.revistas.una.ac.cr/mhsalud
Javier Arturo Hall-López, Paulina Yesica Ochoa-Martínez, Adrián Zamudio Bernal, Ricardo Sánchez León, Luis Gerardo Uriarte Garza, Bartolomé Jesús Almagro, José Moncada-Jiménez, Pedro Sáenz-López Buñuel

\section{Agradecimientos}

Por el financiamiento, a la Coordinación de Posgrado en Investigación de la Universidad Autónoma de Baja California, quienes aportaron los fondos por medio de la 18va convocatoria interna de investigación con el protocolo con clave 149/2/C/9/18.

A la Convocatoria de Becas Movilidad Académica 2015 y 2016, por permitir realizar estancias de investigación a los académicos de la Universidad de Huelva y la Universidad de Costa Rica en la Universidad Autónoma de Baja California y realizar acciones de cooperación en este proyecto.

\section{Referencias}

Bacardi-Gascon, M., Perez-Morales, M. E., y Jimenez-Cruz, A. (2012). A six month randomized school intervention and an 18-month follow-up intervention to prevent childhood obesity in Mexican elementary schools. Nutricion Hospitalaria, 27(3),755-762.

Blanchard, C., Shilton, T. y Bull, F. (2013). Global Advocacy for Physical Activity (GAPA): global leadership towards a raised profile. Glob Health Promotion, 20(4), 113-121.

Banville, D. (2006). Analysis of exchanges between novice and cooperating teachers during internships using the NCATE/NASPE standards for teacher preparation in physical education as guidelines. Research Quarterly for Exercise and Sport, 77(2), 208-221.

Broyles, S., Katzmarzyk, P. T., Srinivasan, S. R., Chen, W., Bouchard, C., Freedman, D.S. y Berenson, G. S. (2010) The pediatric obesity epidemic continues unabated in Bogalusa, Louisiana. Pediatrics, 125(5), 900-905.

Ebbeling, C. B. y Ludwig, D. S. (2010). Pediatric obesity prevention initiatives: more questions than answers. Archives of Pediatrics and Adolescent Medicine, 164(11),1067-1069.

Elizondo-Montemayor, L., Gutierrez, N. G., Moreno, D. M., Martinez, U., Tamargo, D. y Trevino, M. (2013) School-based individualized lifestyle intervention decreases obesity and the metabolic syndrome in Mexican children. Journal of Human Nutrition and Dietetics, 26(1), 82-89.

Instituto Nacional de Salud Pública (INSP).(2012). Encuesta Nacional de Salud y Nutrición 2012 (ENSANUT). Recuperado de http://ensanut.insp.mx/

Flores-Moreno, P., Salazar, C., Gómez-Figueroa, J., Barreto-Villa, Y., Valdovinos-González, O., Vicente-Rivera, J., y Del Río-Valdivia, J. (2017). Medición del tiempo efectivo de la clase de educación física y su impacto en el gasto calórico en escolares de nivel primaria del municipio de Colima, México. Sportis, 3, 34-49. 
Revista en Ciencias del Movimiento Humano y Salud

URL: http://www.revistas.una.ac.cr/mhsalud
Javier Arturo Hall-López, Paulina Yesica Ochoa-Martinez, Adrián Zamudio Bernal, Ricardo Sánchez León, Luis Gerardo Uriarte Garza, Bartolomé Jesús Almagro, José Moncada-Jiménez, Pedro Sáenz-López Buñuel

Gharib, H., Galavíz, K., Lee, R., Safdie, M., Tolentino, L., Barquera. S. y Lévesque, L. (2015). The Influence of Physical Education Lesson Context and Teacher Behaviour on Student Physical Activity in Mexico. RETOS, 28, 160-164.

Gonzalez, A., Boyle, M. H., Georgiades, K., Duncan, L., Atkinson, L. R. y MacMillan, H. L. (2012). Childhood and family influences on body mass index in early adulthood: findings from the Ontario Child Health Study. BMC Public Health, 12,755.

Hall López, J. A., Ochoa Martínez, P. Y., Borbón Román, J. C. y Monreal Ortíz, L. R. (2013). Prevalencia de Porcentaje de Grasa Corporal, Obesidad Abdominal y Estado Nutricional en una Escuela Primaria de Mexicali Baja California México. International Journal of Morphology, 31(4), 1269-1275.

Hall-López, J, A., Ochoa-Martínez, P, Y., González-Terrazas, J, C., y González-Ramírez, J. R. (2017). Duración, intensidad y contexto de clases de Educación Física impartidas por profesores y estudiantes de licenciatura. Sportis, 3, 577-597.

Hall-López, J, A., Ochoa-Martínez, P, Y., Zuñiga, R., Monreal, L, R., y Sáenz- López, P. (2017). Moderate-to-vigorous physical activity during recess and physical education among mexican elementary school students. RETOS, 31, 137-139.

Huang, S. Y., Hogg, J., Zandieh, S. y Bostwick, S. B. (2012) A ballroom dance classroom program promotes moderate to vigorous physical activity in elementary school children. American Journal of Health Promotion, 26(3), 160-165.

Janssen, I., Medina, C., Pedroza, A. y Barquera, S. (2012). Screen time in Mexican children: findings from the 2012 National Health and Nutrition Survey (ENSANUT 2012). Salud Pública de México, 55(5), 484-491.

Jennings-Aburto N., Nava F., Bonvecchio A., Safdie M., González-Casanova I., Gust T. y Rivera J. (2009). Physical activity during the school day in public primary schools in Mexico City. Salud Publica Mex. 51(2), 141-147.

Kelder, S. H., Mitchell, P. D., McKenzie, T. L., Derby, C., Strikmiller, P. K., Luepker, R. V. y Stone, E. J. (2003). Long-term implementation of the CATCH physical education program. Health Education \& Behavior, 30(4), 463-475.

Kelly, A. S., Barlow, S. E., Rao, G., Inge, T. H., Hayman, L. L., Steinberger, J. y Council on Clinical, C. (2013). Severe obesity in children and adolescents: identification, associated health risks, and treatment approaches: a scientific statement from the American Heart Association. Circulation,128(15), 1689-1712. 
Revista en Ciencias del Movimiento Humano y Salud

URL: http://www.revistas.una.ac.cr/mhsalud
Javier Arturo Hall-López, Paulina Yesica Ochoa-Martínez, Adrián Zamudio Bernal, Ricardo Sánchez León, Luis Gerardo Uriarte Garza, Bartolomé Jesús Almagro, José Moncada-Jiménez, Pedro Sáenz-López Buñuel

Léger, L.; Lambert, J.; Goulet, A.; Rowan C. y Dinelle, Y. [Aerobic capacity of 6 to 17-year-old Quebecois--20 meter shuttle run test with 1 minute stages].(1984). Canadian journal of applied sport sciences, 9(2), 64-9.

Lizana Arce, P. J., Almagià Flores, A. A., Simpson Lelievre, M. C., Olivares Barraza, R., Binvignat Gutiérrez, O., Ivanovic Marincovich, D. y Berral de la Rosa, F. J. (2011). Inconsistency Between the Body Fat Percentages Estimated Through Anthropometric Measurements and Manual Bioimpedance in Children and Adolescents. International Journal of Morphology, 29(4), 1364-1369.

Marfell-Jones, M.; Olds, T., Stewart, A. y Carter, L. (2006) International standards for anthropometric assessment. Potchefstroom, South Africa, ISAK.

Mckenzie, T. L.; Sallis, J. F. y Nader, P. R. (1992). Sofit - System for Observing Fitness Instruction Time. Journal of Teaching in Physical Education, 11(2), 195-205.

National Association for Sport and Physical Education (NASPE). (2009). National standards and guidelines for physical education teacher education (3era ed.). Reston, VA: Autor.

Osganian, S. K., Parcel, G. S. y Stone, E. J. (2003). Institutionalization of a school health promotion program: background and rationale of the CATCH-ON study. Health Education \& Behavior, 30(4), 410-417.

Organización Mundial de la Salud (OMS). (s.f.). Global Strategy on Diet, Physical Activity and Health. Physical activity and young people. Recuperado de http://www.who.int/ dietphysicalactivity/factsheet_young_people/en/

Perichart-Perera, O., Balas-Nakash., M., Ortiz-Rodríguez, V., Morán-Zenteno, J. A., GuerreroOrtiz, J. L. y Vadillo-Ortega, F. (2008). A program to improve some cardiovascular risk factors in Mexican school age children. Salud Publica de Mexico, 50(3), 218-26.

Pérez Bonilla, A. M. (2009). Impacto de la clase de Educación Física sobre la actividad moderada y vigorosa en niños de primaria. Rev Mex Cult Fis, 1(1)150-172.

Perry, C. L., Stone, E. J.; Parcel, G. S.; Ellison, R. C.; Nader, P. R.; Webber, L. S. y Luepker, R. V. (1990). School-based cardiovascular health promotion: the child and adolescent trial for cardiovascular health (CATCH). Journal of School Health, 60(8), 406-413.

Rank, M., Siegrist, M., Wilks, D. C., Langhof, H., Wolfarth, B., Haller, B. y Halle, M. (2013). The cardio-metabolic risk of moderate and severe obesity in children and adolescents. Journal of Pediatrics, 163(1), 137-142.

Ruiz, J. R., Castro-Pinero, J., Espana-Romero, V., Artero, E. G., Ortega, F. B., Cuenca, M. M. y Castillo, M. J. (2011). Field-based fitness assessment in young people: the ALPHA 

Adrián Zamudio Bernal, Ricardo Sánchez León,

health-related fitness test battery for children and adolescents. British Journal of Sports Medicine, 45(6), 518-524.

Safdie, M., Jennings-Aburto, N., Lévesque, L., Janssen, I.,Campirano-Núñez, F., López-Olmedo, N., Aburto, T. y Rivera J. A. (2013). Impact of a school-based intervention program on obesity risk factors in Mexican children. Salud Publica de Mex, 55(3), 374-87.

Slaughter, M. H., Lohman, T. G., Boileau, R. A., Horswill, C. A., Stillman, R. J., Van Loan, M. D. y Bemben, D. A. (1988). Skinfold equations for estimation of body fatness in children and youth. Human Biology, 60(5), 709-723.

Stanley, J., Peake, J. M. y Buchheit, M. (2013). Cardiac parasympathetic reactivation following exercise: implications for training prescription. Sports Medicine, 43(12), 1259-1277.

Szklo M, Nieto J. (2007). Epidemiology: beyond the basics (2nd ed.) Jones \& Bartlett Learning.

Tailor, A. M., Peeters, P. H., Norat, T.,Vineis, P. y Romaguera, D. (2010). An update on the prevalence of the metabolic syndrome in children and adolescents. International Journal of Pediatric Obesity, 5(3), 202-213.

Welk, G. J., De Saint-Maurice Maduro, P. F., Laurson, K. R. y Brown, D. D. (2011). Field evaluation of the new FITNESSGRAM(R) criterion-referenced standards. American Journal of Preventive Medicine, 41(2), 131-142.

Fecha de recibo: 13 de marzo del 2017

Fecha de corrección: 18 de mayo del 2017

Fecha de aceptación: 15 de junio del 2017

Fecha de publicación: 30 de agosto del 2017 